\title{
Finite Element Analysis based Optimization of Magnetic Adhesion Module for Concrete Wall Climbing Robot
}

\author{
MD Omar faruq Howlader \\ Robotics and NDT Research Center \\ London South Bank University \\ London, UK
}

\author{
Traiq Pervez Sattar \\ Robotics and NDT Research Center \\ London South Bank University \\ London, UK
}

\begin{abstract}
Wall climbing robot can provide easier accessibility to tall structures for Non Destructive Testing (NDT) and improve working environments of human operators. However, existing adhesion mechanism for climbing robots such as vortex, electromagnet etc. are still at development stage and offer no feasible adhesion mechanism. As a result, few practical products have been developed for reinforced concrete surfaces, though wall-climbing robots have been researched for many years. This paper proposes a novel magnetic adhesion mechanism for wallclimbing robot for reinforced concrete surface. Mechanical design parameters such as distance between magnets, the yoke thickness, and magnet arrangements have been investigated by Finite Element Analysis (FEA). The adhesion module can be attached under the chassis of a prototype robot. The magnetic flux can penetrate maximum concrete cover of $30 \mathrm{~mm}$ and attain adhesion force of $\mathbf{1 2 1 . 2 6} \mathrm{N}$. The prototype provides high Forceto-Weight ratio compared to other reported permanent magnet based robotic systems. Both experiment and simulation results prove that the magnetic adhesion mechanism can generate efficient adhesion force for the climbing robot to operate on vertical reinforced concrete structures.
\end{abstract}

Keywords-Finite Element Analysis (FEA); Magnetic Adhesion System; Non Destructive Testing (NDT); Wall Climbing Robot

\section{INTRODUCTION}

This research aims to develop a novel adhesion mechanism for concrete wall climbing robot. In this paper, the design parameters and development of a prototype robot are reported. Many of the structures such as high-rise buildings, bridges, dams, complex nuclear power plants etc. are constructed using concrete because of its high strength and durability. Over the lifetime, natural phenomenon like moisture and chlorides present in the atmosphere cause the concrete structures to lose their strength and that induce structural faults like reinforcement bar corrosion, cracking, and delamination of the concrete surface. Currently manual Non Destructive Testing (NDT) using handheld devices are used to detect faults in concrete surface and overall structural integrity. Figure 1 shows the scenario of an operator carrying out manual NDT of a $92 \mathrm{~m}$ tall concrete chimney using handheld ground penetrating radar.

Likewise, current method of carrying out NDT process is manual and less efficient as access to test sites on these structures is obtained by constructing scaffolding or abseiling down from the top. Operators deal with hazardous environments such as nuclear industrial environments, working at high altitude, and limited manoeuvrability [2]. In terms of safety, cost and efficiency, climbing robots can offer reliable performance and access to large structures in hazardous environments that may not be accessible to human inspectors.
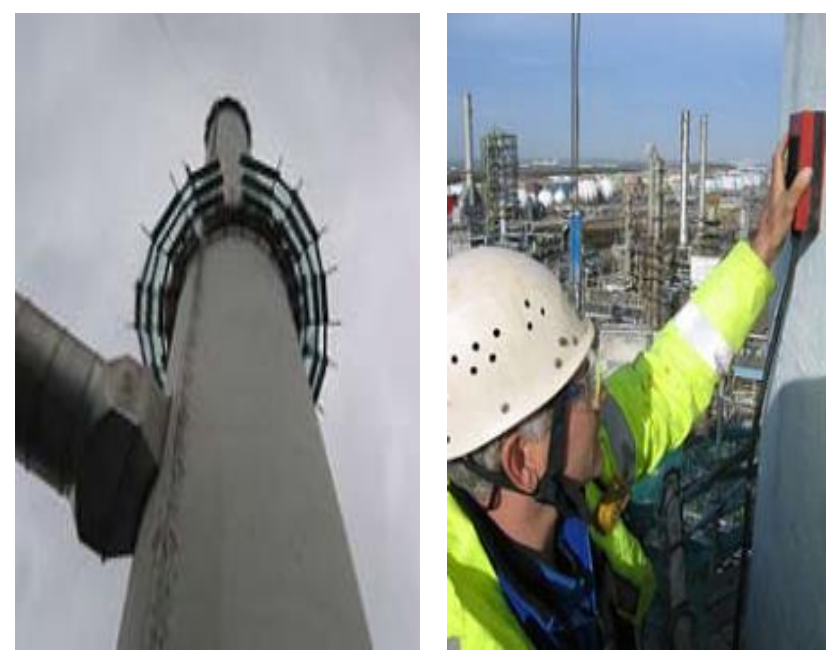

Fig. 1. Manual NDT of tall concrete structure by erecting scaffolding [1]

The proposed prototype robot discussed in this paper is designed to provide robotic platform to attach variety of NDT equipment for concrete wall inspection. The general requirements of the robot mainly include:

a) The robot should have climbing capability of vertical steel surfaces of up to $100 \mathrm{~m}$ high and curved surfaces where the minimum diameter is down to $10 \mathrm{~m}$ which is common for concrete based nuclear power plants.

b) Keep the robot weight to a minimum as possible. Should the robot fail for any reason, the impact of a heavy robot would cause damage to plant equipment and surroundings.

c) Retain a minimum payload capacity of 2-5 $\mathrm{kg}$ for mounting NDT equipment.

d) It should be able to make floor to wall transition and vice versa with a maximum angle of $90^{\circ}$. 
This paper proposes a permanent magnet based adhesion system for concrete surface climbing robot that is distinctive compared to other systems found in the literature. The permanent magnets are arranged in a special way that the magnetic flux is concentrated and magnified to couple with reinforcement bars (rebars) buried under the concrete. The paper consists of the following sections: section II gives an overview of the existing climbing robots. Section III describes the construction method of concrete structures. Dynamic force analysis to establish climbing force requirement and optimization of magnetic adhesion module using Finite Element Analysis (FEA) are given in section IV and $\mathrm{V}$ respectively. Section VI presents the experiment setup for adhesion performance verification with comparison of this proposed system with other available systems in section VII and finally, the paper concludes with a brief summary and recommendation in section VIII.

\section{CURRENT StATE OF Climbing Robot}

Most important considerations in developing wallclimbing robots are its mechanical design, locomotion and adhesion mechanism. Climbing robots need to have the same locomotion mechanism as mobile robots however, the adhesion mechanism significantly affects the system reliability, payload ability, and power consumption of robots. That makes it more challenging to develop a climbing robot than a mobile robot.

To overcome the limiting factors of manual NDT process, application of special climbing robots has been seen in fields such as steel oil tank inspection, weld line inspection, steel pipe structure inspection [3]. There are number of adhesion techniques for climbing robot such as vacuum suction cups, magnets, negative pressure vortex that could be found in the literature. Permanent magnets have been used as the primary adhesion method in designing ferrous wall-climbing robots. Magnetic wheel-type [4], track-type mechanisms [5], legged robots with magnetic feet [6] are used for robot inspecting complex shaped structures. The benefit of wheel-type locomotion is that it can move flexibly with a small contact surface between the wheels and the wall. Therefore, the robot's energy use ratio is low. The track-type mechanism has a larger contact area and can generate higher attraction force however, it is hard to change directions. A non-contact adhesion mechanism has been proposed in [7]. It mounts arrays of magnets under the chassis of the robot. There is a gap between the magnet and the wall surface. However, the effect of multiple magnet layer and the distance between the magnets on total adhesion force are not investigated there. Reference [8] also proposes permanent magnet based system. It investigates the effect of air gap between multiple magnets and the steel surface, the effect of distance between the magnets, effect of magnet dimension on overall adhesion force have not been investigated though. Suction cups and pneumatic adhesion are other popular methods of adhesion. Robots using more traditional suction mechanism are the ROBICEN [9], NINJA-II [10], ROBIN [11]. The Clarifying Climber III [12] is a robot that works by creating negative pressure called vortex between the surface and the robot body. Another robotic system developed for the purpose of concrete NDT is Tile-Wall robot as shown in fig. 2. This includes a mobile module which carries the sensors and NDT devices, a ground platform and a roof platform which work together to form a vertical conveyor belt system to slide the robot up and down [13].

Even though such system solves the difficulty of climbing vertically but it still needs the conveyor system to be installed on the roof and ground. This feature makes this system ineffective in case of very tall structures.

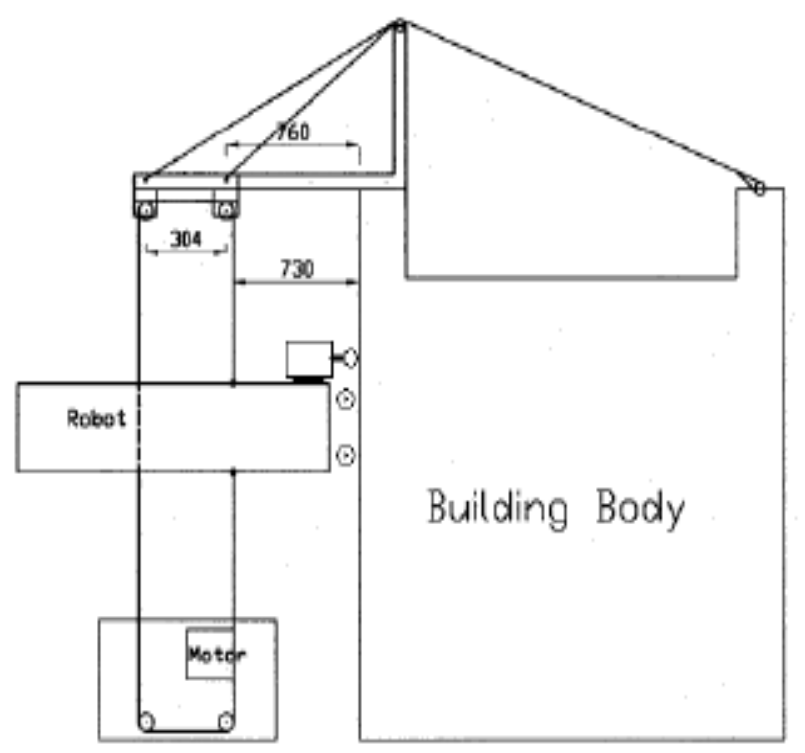

Fig. 2. Block diagram of Tile-Wall robot system [13]

Developing climbing robot for reinforced concrete surface presents a new aspect in adhesion technique as adhesion principles of vortex and electro adhesion are still in development stage and not completely understood as well as suction cups, which operation is limited to smooth surfaces. Moreover, tracked vehicles having two degrees of freedom are less maneuverable. As a result, few practical products have been developed for reinforced concrete surfaces, though wallclimbing robots have been researched for many years.

\section{CONSTRUCtion Method OF CONCRETE StRUCTURES}

Permanent magnets can establish magnetic coupling with ferromagnetic materials present within the magnetic field. All safety critical concrete structures are reinforced with steel rebars. Steel is highly ferromagnetic material therefore, means to establish magnetic adhesion between rebars buried under concrete and magnets could provide a simple, low-energy adhesion mechanism. However, the main challenge when adhering to such structures is to shape the magnetic flux to flow into the concrete as deeply as possible. Hence, it is important to investigate the construction methods of safety critical concrete structures.

Concrete is a brittle material. It has strong compression force but cannot withstand strong tensile stress [14]. As a consequent, cracking on a non-reinforced concrete plane could easily occur if excessive force if applied by external loading as shown in fig. 3. Therefore, raw concrete is reinforced with strong materials such as steel to counter the tensile forces resulting from induced loading. Dense meshing of steel rebars on the vertical wall of a nuclear power plant is shown in fig. 4 . 
However, a correct rebar positioning is critical. For instance, if rebars are located near the bottom surface of a concrete slab and if the surface is subjected to excessive force applied by the load then this will lead to appearance of cracks on the bottom plate as in fig. 5 and must follow a standard to ensure overall structural strength.

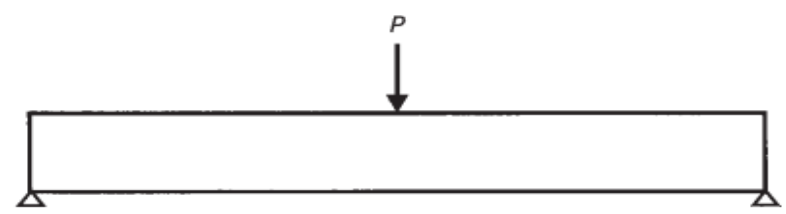

(a)

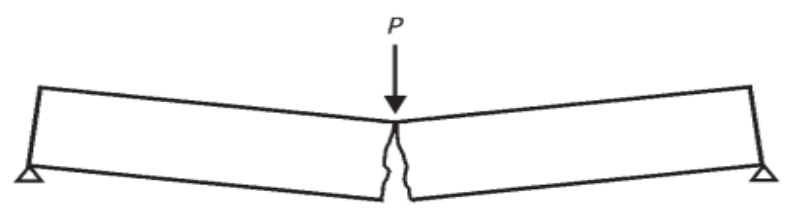

(b)

Fig. 3. Cracks on a concrete plane (a) Bending Force $<$ Tensile, (b) Bending Force > Tensile [14]

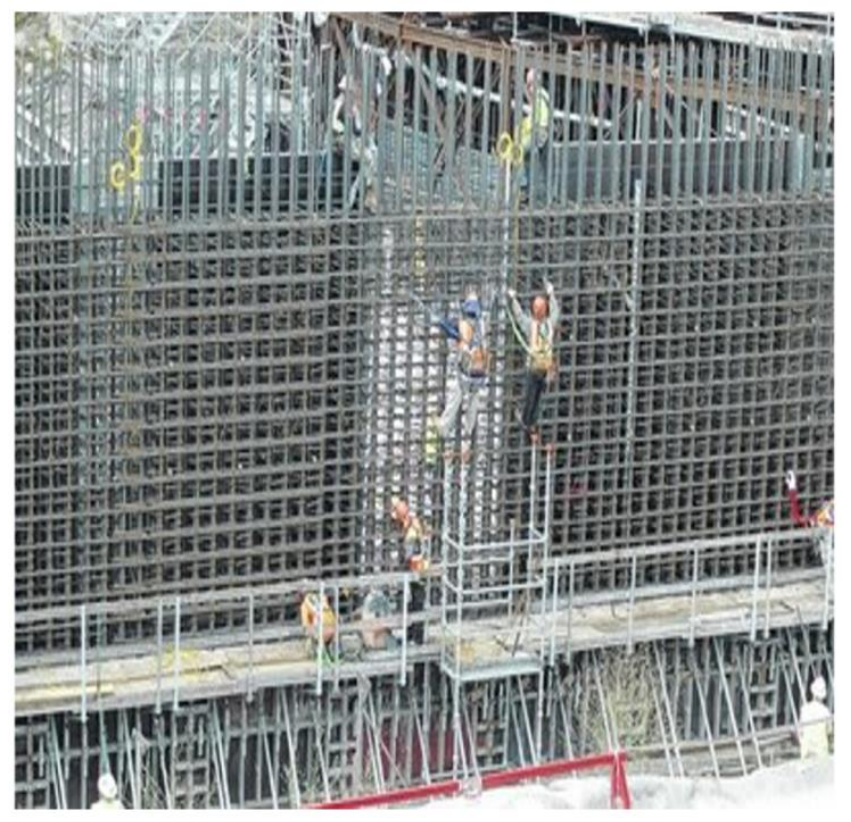

Fig. 4. Proposed workspace for the concrete wall-climbing robot

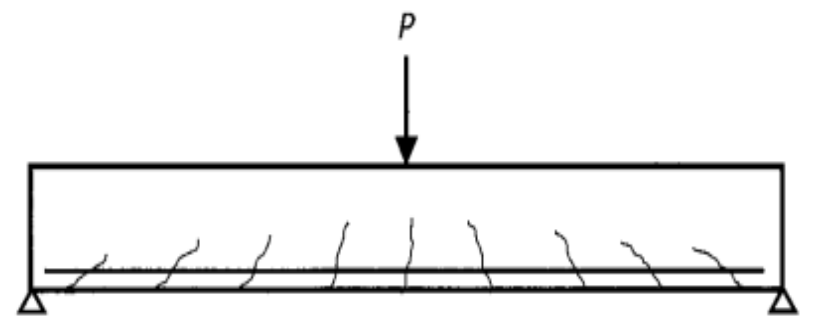

Fig. 5. Appearance of cracks due to inaccurate rebar positioning [14]

The standard applicable in UK that determines the minimum concrete cover requirement is BS 850 and Eurocode 2 [15].
In Eurocode 2, different structures are categorized in different classes such as buildings and power plants are XC3 and XC4 class structures as they are exposed to cyclic wet, dry and moderate humid conditions whereas bridge columns that are submerged in water are $\mathrm{XC} 1$ class. The nominal concrete cover is determined based on the type of environmental exposure, concrete quality, and intended working life of the structure. Therefore, the following measurement should apply for nominal concrete cover where an allowance of $10 \mathrm{~mm}$ in design deviation is introduced.

$$
\mathrm{C}_{\text {nominal }}=\mathrm{C}_{\text {minimum }}+10 \mathrm{~mm}
$$

The nominal cover requirement for atmospheric exposure of 50 years working life extracted from BS 850 is given in table I.

TABLE I. NOMINAL CONCRETE COVER FOR DIFFERENT STRUCTURES BASED ON BS 850

\begin{tabular}{|l|l|l|}
\hline Structures & $\begin{array}{l}\text { Minimum range, } \\
\text { mm (Cement } \\
\text { quality } \\
\text { dependent) }\end{array}$ & $\begin{array}{l}\text { Nominal range, } \\
\text { mm (Cement } \\
\text { quality } \\
\text { dependent) }\end{array}$ \\
\hline $\begin{array}{l}\text { Buildings (Vertical } \\
\text { elements exposed to rain } \\
\text { and snow) }\end{array}$ & $25-35$ & $30-45$ \\
\hline Bridge Columns & $25-35$ & $30-45$ \\
\hline Dam & $25-50$ & $35-60$ \\
\hline Nuclear Power Plant & $25-35$ & $30-45$ \\
\hline
\end{tabular}

The rebars diameter can vary from $8-55 \mathrm{~mm}$. The primary requirement of the adhesion module is to penetrate magnetic flux to a maximum of $60 \mathrm{~mm}$ depth to couple with the rebars and generate sufficient adhesion force to ascend on the vertical wall carrying the payload.

\section{DYNAMIC FORCE ANALYSIS}

The ideal operation of a climbing robot involves vertical climbing, transitioning and climbing angled slopes. A force analysis could provide insights into stability of the climbing robot and determine minimum adhesion force requirement to avoid sliding and roll-over failure.

\section{A. Sliding avoidance}

To understand the forces acting on a robot, consider the forces acting on a robot resting on an inclined plane as shown in fig. 6. If robot weight is $=\mathrm{W}$, distance of front and back wheel center $=\mathrm{L}$, height of the center of gravity $=\mathrm{d}$, acceleration of the robot $=\mathrm{a}$, the coefficient of surface friction $=\mu$, slope of the inclined surface $=\theta$.

$$
\begin{gathered}
\sum F_{x}=W \sin \theta-\mu N \\
N=\frac{W \sin \theta}{\mu} \\
W \cos \theta+F_{a}=\frac{W \sin \theta}{\mu}
\end{gathered}
$$

Therefore, for the robot to avoid slipping, required adhesion force should be

$$
F_{a}>\frac{W \sin \theta}{\mu}-W \cos \theta
$$



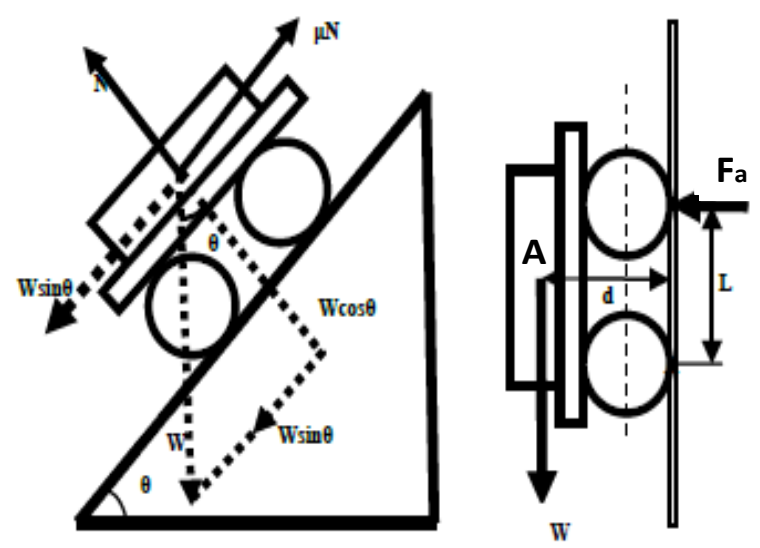

Fig. 6. Free body diagram of robot moving on an angled surface

If the robot is operating on a completely vertical surface then $\Theta=90^{\circ}$ and equation stands as

$$
F_{a}>\frac{W}{\mu}
$$

\section{B. Capsizing avoidance}

The torque induced by the front set of wheels should be greater than that induced by the robot's weight. So, by taking moment about point A from fig. 6 ,

$$
\begin{gathered}
\sum M=W \times d+F_{a} \times L=0 \\
F_{a}=-\frac{W \times d}{L}
\end{gathered}
$$

Here negative sign denotes the force direction. To avoid capsizing, the adhesion force should be,

$$
F_{a}>\frac{W \times d}{L}
$$

In order to avoid slipping, the center of gravity of the robot should be as close to ground as possible and the distance between the wheels should be large enough. Moreover, increasing the coefficient of surface friction and reducing the robot weight will ensure capsizing avoidance. In addition, the calculations suggest that as long as the robot can avoid slipping, it can avoid capsizing as the force required to avoid capsizing is significantly lower than slipping.

\section{DESIGN Optimization OF AMGNETIC AdHESION MODULE}

The main challenge when adhering to concrete structure using permanent magnet is to shape the magnetic flux to flow into the concrete as deeply as possible to couple with rebars. FEA could be used to investigate the magnetic flux propagation behavior and also measure the resultant adhesion force.

Mathematical model of FEA based magneto-static simulation

Magneto-static models are better understood by Maxwell's equations. If magnetic field intensity is $H$, magnetic induction is $B$ then according to Maxwell equation the relations between them in a static magnetic field are [16]:

$$
\nabla \times \mathrm{H}=\mathrm{J}
$$

$$
\begin{aligned}
& \nabla . \mathrm{B}=0 \\
& \mathrm{~B}=\mu \mathrm{H} \\
& \mathrm{J}=\sigma \mathrm{E}
\end{aligned}
$$

Here, $J$ is current density, $\mu$ is dielectric permeability, $E$ is electric field intensity, and $\sigma$ the conductivity of the medium. The magnetic permeability of ferrous materials such as steel and iron is non-linear and in reverse relation with the magnetic field intensity, $H$. The magnetic field generated the proposed adhesion unit is regarded as static and the electromagnetic field generates a uniform magnetic field effect in this situation. The field vectors and source vectors in Eq. (2) - Eq. (8) are all space co-ordinate functions and do not change with respect with time.

The double rotation equation of the equivalent vector magnetic potential function is:

Where, $A$ is a vector

$$
\nabla^{2} \times \mathrm{A}=\mu \mathrm{J}
$$

In a three-dimensional co-ordinate system, Eq. (6) can be translated into

$$
\begin{aligned}
& \nabla^{2} \times A_{x}=\frac{\partial^{2} A_{x}}{\partial x^{2}}+\frac{\partial^{2} A_{x}}{\partial y^{2}}+\frac{\partial^{2} A_{x}}{\partial z^{2}}=-\mu J_{x} \\
& \nabla^{2} \times A_{y}=\frac{\partial^{2} A_{y}}{\partial x^{2}}+\frac{\partial^{2} A_{y}}{\partial y^{2}}+\frac{\partial^{2} A_{y}}{\partial z^{2}}=-\mu J_{y} \\
& \nabla^{2} \times A_{z}=\frac{\partial^{2} A_{z}}{\partial x^{2}}+\frac{\partial^{2} A_{z}}{\partial y^{2}}+\frac{\partial^{2} A_{z}}{\partial z^{2}}=-\mu J_{z}
\end{aligned}
$$

The FEA divides the solution area into small areas by using tetrahedral meshing method and calculates the resultant force as an integral of Maxwell's surface stress tensor equation as below [17],

$$
n_{1} T_{2}=-\frac{1}{2}(H \cdot B) n_{1}+\left(n_{1} \cdot H\right) B^{T}
$$

Where, $\mathrm{n}_{1}$ is the boundary normal pointing out from the rebars and $\mathrm{T}_{2}$ is the stress tensor of air.

\section{A. Optimization of magnetic adhesion system}

To understand the design criteria of an adhesion module for reinforced concrete structures, 3D model simulations are carried out using industry standard software Comsol Multiphysics. Here parameters such as distance between multiple magnets, implementation of flux concentrator, variable concrete cover etc. are investigated to determine their effect on resultant adhesion force, $\mathrm{F}_{\mathrm{a}}$. The main properties of the materials used in simulations are listed in table II.

TABLE II. MAIN PROPERTIES OF NDFEB N42

\begin{tabular}{|c|c|}
\hline Properties & Value \\
\hline Magnetic induction intensity $\mathrm{B}_{\mathrm{r}}(\mathrm{T})$ & 1.31 \\
\hline Coercive force $\mathrm{H}_{\mathrm{cb}}(\mathrm{KA} / \mathrm{m})$ & 915 \\
\hline Intrinsic coercive force $\mathrm{H}_{\mathrm{ci}}(\mathrm{KA} / \mathrm{m})$ & 955 \\
\hline Magnetic energy product $\mathrm{HB}\left(\mathrm{KJ} / \mathrm{m}^{3}\right)$ & 318 \\
\hline Relative permeability $\left(\mu_{\mathrm{r}}\right)$ & $1.068-1.113$ \\
\hline Relative permeability of yoke $\left(\mu_{\mathrm{r}}\right)$ & 5000 \\
\hline Relative permeability of steel concrete \\
surface $(\mu \mathrm{r})$ & 1 \\
\hline Relative permeability of steel rebar $(\mu \mathrm{r})$ & 1500 \\
\hline
\end{tabular}


The primary focus here is to achieve maximum adhesion force to support the payload by using minimum number of magnets. Therefore, keeping the robot's net weight, $\mathrm{W}$ to a minimum will help to increase the performance of the adhesion module. So, the value of magnetic force ratio, $\eta$ directs the performance of adhesion unit where,

$$
\eta=\frac{F_{a}}{W}
$$

1) Effects of distance between multiple magnets

If two magnets are placed close to each other, then the distance between them affects the distribution of magnetic flux and thus the total adhesion force. To evaluate this scenario, two neodymium grade N42 magnets are attached to a grey cast iron ( $97 \%$ pure) plate called "yoke" with reversed polarity. Dimensions used are magnet length, $M_{l}=50 \mathrm{~mm}$, width, $M_{w}=50$ $\mathrm{mm}$, thickness, $\mathrm{M}_{\mathrm{t}}=12 \mathrm{~mm}$, rebar diameter, $\mathrm{R}_{\mathrm{d}}=12 \mathrm{~mm}$, yoke thickness, $Y_{t}=10 \mathrm{~mm}$; concrete cover, $C_{c}=30 \mathrm{~mm}$. The distance between the two magnets are varied from $10 \mathrm{~mm}$ to $150 \mathrm{~mm}$ and the magnetic flux density norms are observed from simulations. The model setup is given in fig. 7 .

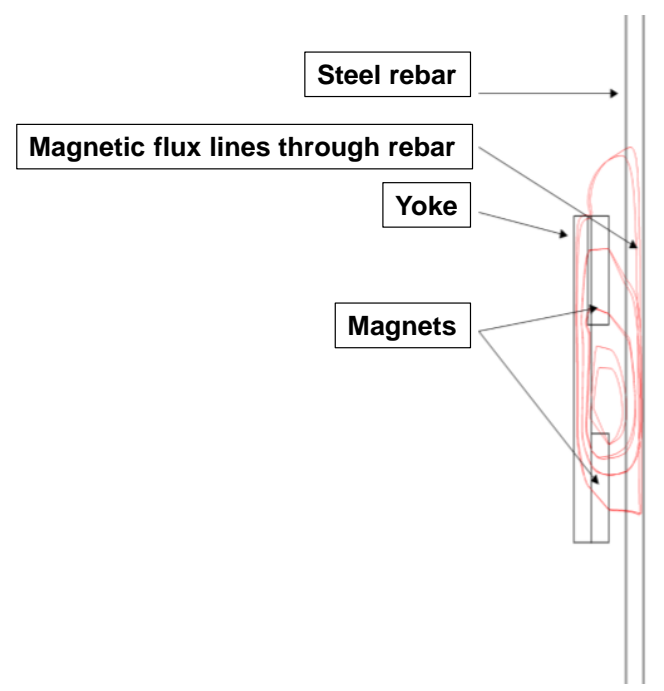

Fig. 7. Distribution of magnetic flux lines in the magnetic circuit travelling from North Pole of one magnet to South Pole of another magnet

The color graphs in fig. 8 represent the magnetic flux concentration norm along the rebar length. It is observed that as the distance between the magnets is increased from $10 \mathrm{~mm}$, the flux concentration area increases shown by the red hotspot on the rebar.

As two magnets are closer to each other, the resultant magnetic attraction area is small. But as the distance increases to an optimum level of 50-60 mm, magnetic flux leaving North Pole of one magnet have to travel longer to couple with South Pole of another magnet to complete the magnetic circuit between two magnets and as a result, total active area of attraction becomes bigger which in turns increases the adhesion force. Bigger red hotspot area in fig. 8(b) denotes the optimum distance between the magnets. At distance greater than $60 \mathrm{~mm}$, magnetic flux density reduces as represented by bigger but low intensity hotspot for $150 \mathrm{~mm}$ magnet distance in fig. 8(c).
According to simulation results in fig. 9, adhesion force increases gradually as the distance between the magnets increases. At $50 \mathrm{~mm}$ distance between magnets, adhesion force is maximum at $64.93 \mathrm{~N}$. This is a $33 \%$ increase compared to adhesion force achieved at $10 \mathrm{~mm}$ distance between magnets. However, adhesion force falls sharply as the distance is increased above $50 \mathrm{~mm}$. At $150 \mathrm{~mm}$ distance, adhesion force reaches as low as $51.6 \mathrm{~N}$. These measurements support the magnetic flux density norm presented.
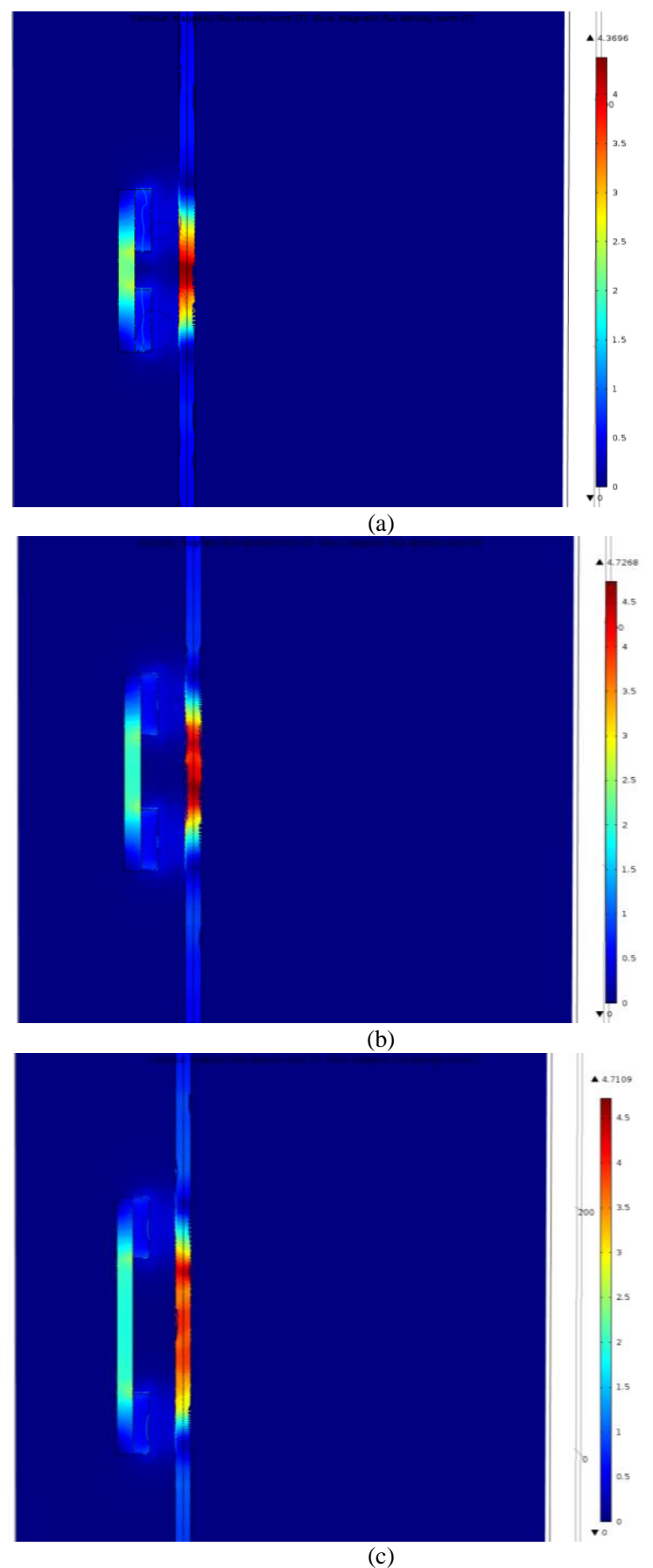

Fig. 8. Magnetic flux concentration norm at different distance between magnets; (a) Distance: 10mm, (b) Distance: 50mm, (c) Distance: $1500 \mathrm{~mm}$ 


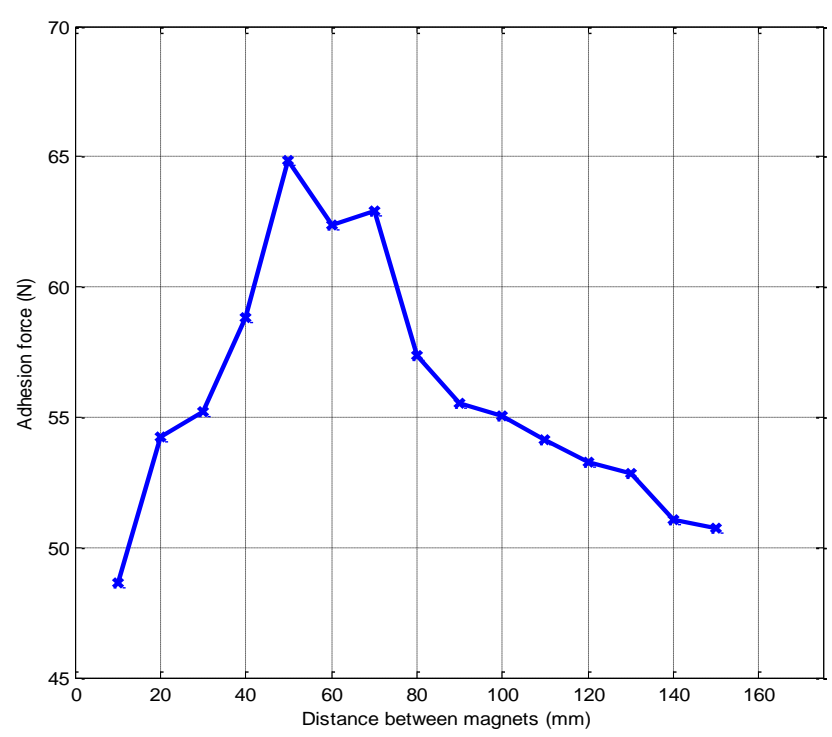

Fig. 9. Simulated adhesion force at different distances between magnets

\section{2) Effects of yoke thickness on adheison force}

Previous simulations show that the use of yoke significantly increases the adhesion force. Using only two magnets placed at $50 \mathrm{~mm}$ distance between them without any yoke results in adhesion force of only $39.34 \mathrm{~N}$ for concrete cover of $30 \mathrm{~mm}$. But implementing a yoke of $5 \mathrm{~mm}$ thickness increases the adhesion force significantly to $53.71 \mathrm{~N}$. Therefore, thickness of yoke plays a critical part to maximize adhesion force. To investigate this parameter further, models with varied yoke thickness from $1 \mathrm{~mm}$ to $40 \mathrm{~mm}$ are simulated. All other parameters are kept the same as previous simulations.

The adhesion force found to be increasing significantly when the yoke thickness is increased from $1 \mathrm{~mm}$ to $20 \mathrm{~mm}$. A yoke of $1 \mathrm{~mm}$ thickness does not have any apparent effect on adhesion force as the flux leakage is higher in all direction as shown in magnetic flux density norm in fig. 10(a).

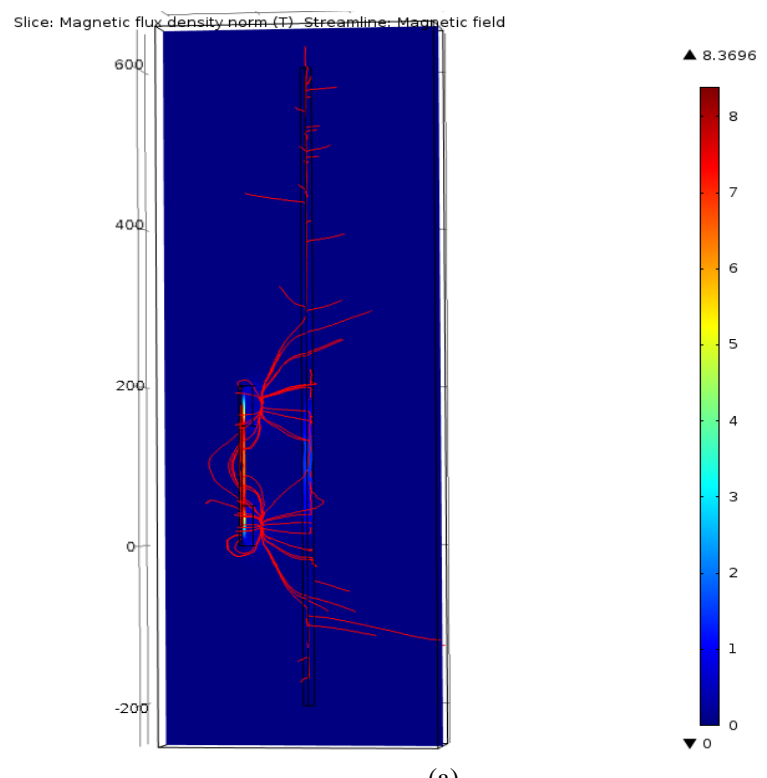

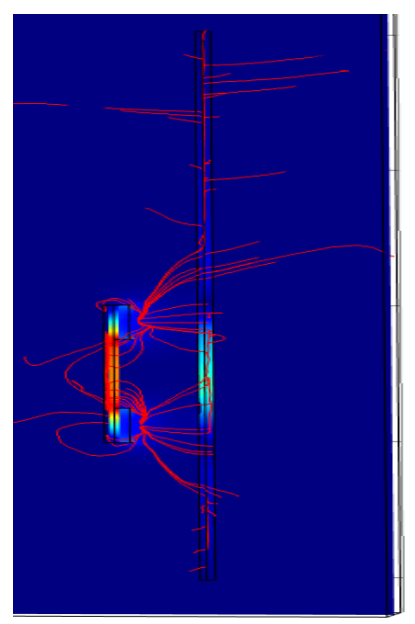

Vol. 6, No. 8, 2015
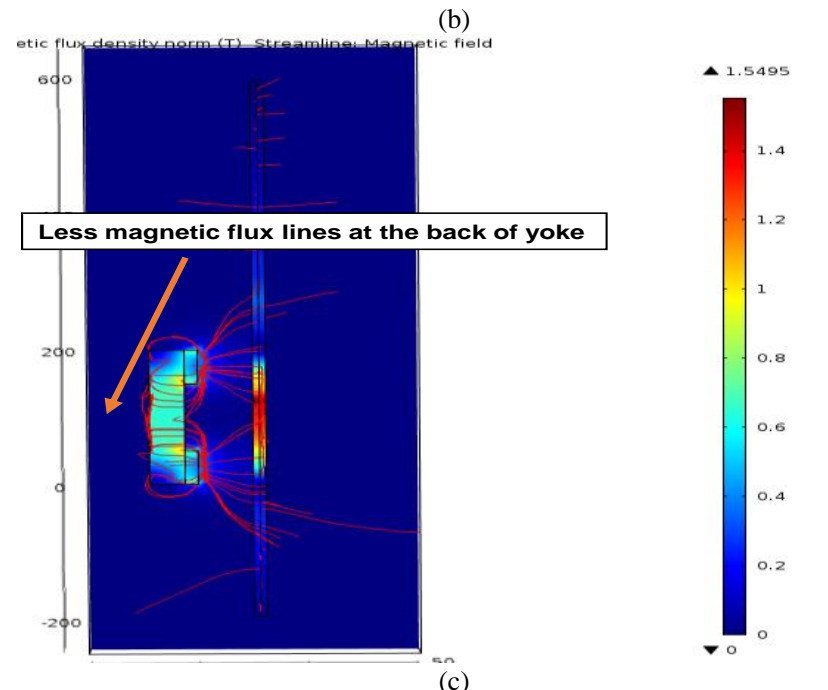

Fig. 10. Magnetic flux density norm at yoke thickness; (a) $1 \mathrm{~mm}$, (b) $5 \mathrm{~mm}$, (c) $20 \mathrm{~mm}$

Any influence of yoke thickness appeared when the thickness is increased to $5 \mathrm{~mm}$, the system achieves magnetic coupling with rebar as presented in fig. 10(b). But at $20 \mathrm{~mm}$ thickness, the flux concentration is at maximum and reaches maximum adhesion force of $65.13 \mathrm{~N}$. Moreover, the magnetic flux leakage is lower in the yoke's opposite surface and more flux lines are concentrated toward the rebar shown as red hotspot in fig. 10(c). According to force against yoke thickness graph in fig. 11, the adhesion force comes to a saturation point of approximately $62 \mathrm{~N}$ at $25 \mathrm{~mm}$ yoke thickness, where further increase in yoke thickness would not have any significant influence on adhesion force. Moreover, the force-to-weight ratio, $\Pi$ of the adhesion module falls sharply because of the added weight by increased yoke thickness. The primary reason for this is that, magnetic flux lines prefer to pass through medium of high permeability. When the yoke thickness exceeds an optimum point (in this case $25 \mathrm{~mm}$ ), the magnetic flux lines prefer to pass through the yoke of high permeability rather than air as in figure 12. Thus, the magnetic coupling with the rebars is minimum to influence the adhesion force. 


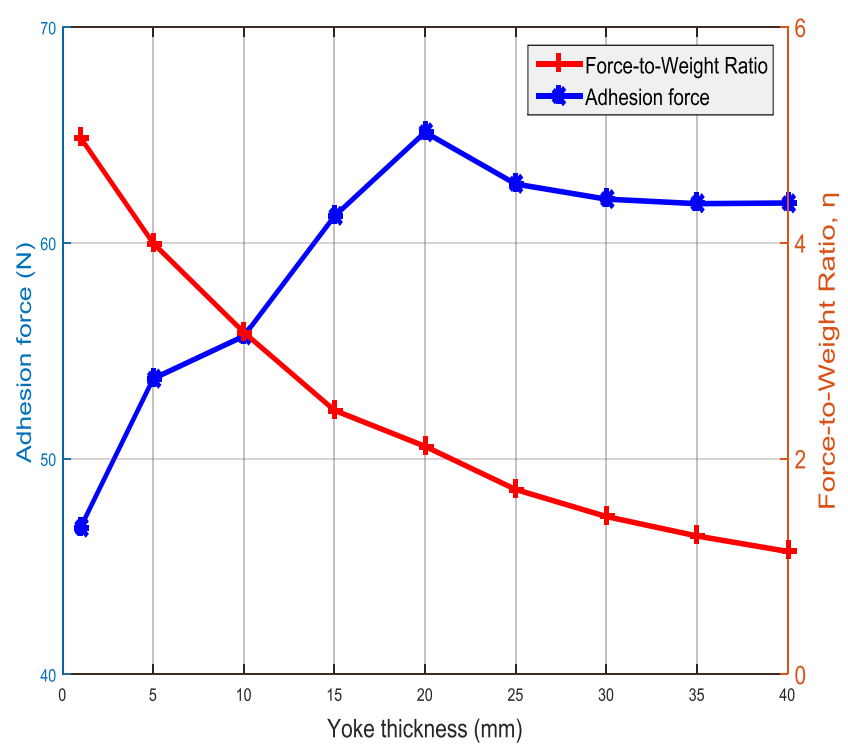

Fig. 11. Adhesion force for yokes with different thicknesses

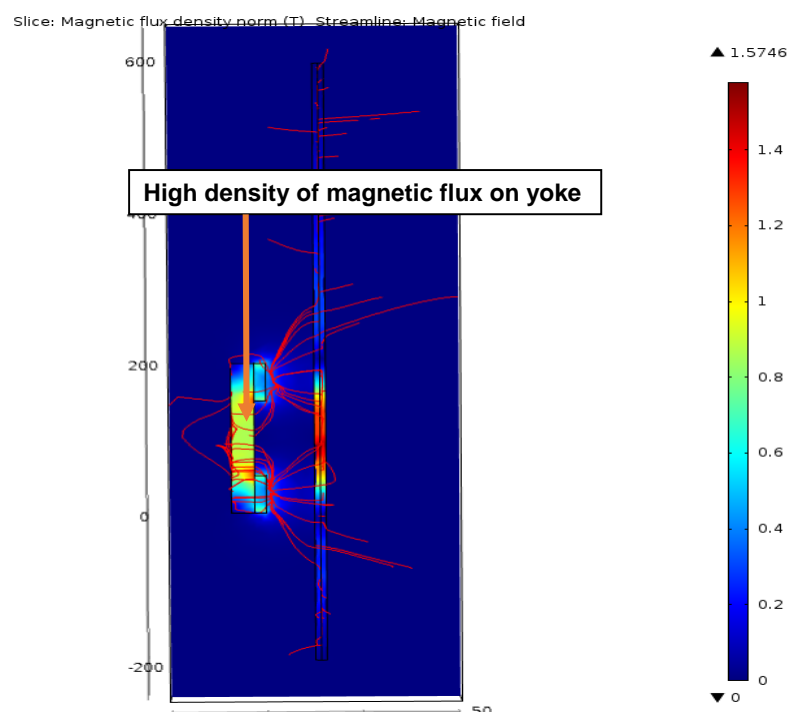

Fig. 12. Density of magnetic flux on a $25 \mathrm{~mm}$ thick yoke

Therefore, the implementation of yoke ensures magnetic flux concentration between the yoke and steel rebars. As a result, attraction force is increased. It also acts as a magnetic shield and reduces the magnetic field strength at the back, so there is less magnetic interference with electronic control circuits.

\section{3) Optimization analysis of magnet arrangement layout}

The influence of the magnet and yoke dimensions on the performance of the adhesion module was analyzed in the previous sections. As the adhesion module is made of multiple magnets so, the coupling between them will influence the adhesion force. For the previous two sets of simulation, NorthSouth configuration using two magnets was implemented. Such configuration essentially creates one magnetic circuit travelling from North Pole of one magnet to South Pole of the other magnet. Yet for deeper penetration of magnetic flux lines, a configuration of North-South-North using three magnets could be considered as fig. 13 .

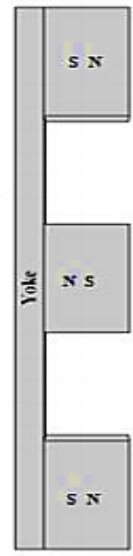

Layout 1

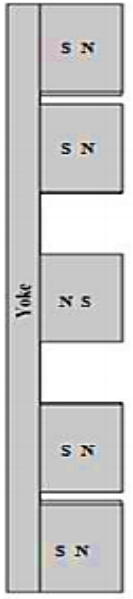

Layout 2
Fig. 13. Proposed N-S-N and N-N-S-N-N magnet arrangement layouts

In this case, adding one magnet on the system will create two separate magnetic circuits and thus might increase the overall adhesion force. Another configuration of North-NorthSouth-North-North could be considered. This arrangement will also effectively create two magnetic circuits however, the magnetic flux lines have to travel further from North Pole to South due to the fact that two identical poles are located near to each other. As a consequent, a deeper dispersion of magnetic flux lines could be accomplished. For simulations, magnet dimension is kept similar to previous cases and yoke thickness of $15 \mathrm{~mm}$ is considered.

Simulation results show that adding one extra magnet into the system greatly increases the module's ability to concentrate the magnetic flux towards the buried rebar and thus can generate greater adhesion force. The maximum adhesion force is measured $113.19 \mathrm{~N}$ for N-S-N layout compared to $65.13 \mathrm{~N}$ in the previous case at $30 \mathrm{~mm}$ concrete cover. According to magnetic flux density norm from Fig. 14, magnetic flux for N$\mathrm{N}-\mathrm{S}-\mathrm{N}-\mathrm{N}$ does not concentrate as uniformly as N-S-N layout. Layout 2 essentially creates two magnetic circuits but as two North Poles are located in close proximity at both ends of the adhesion module, it actually distort the magnetic flux uniformity. Moreover, in case of layout 2, having two same poles closer to each other ensure that magnetic flux lines travel a longer distance to meet the opposite pole compared to layout 1.

However, adhesion force comparison result between two layouts shows that, layout 2 produces higher adhesion force than layout 1 for different concrete cover. At $10 \mathrm{~mm}$ concrete cover, resultant adhesion force is $312.57 \mathrm{~N}$ and $451.33 \mathrm{~N}$ respectively for layout 1 and 2 . But as the concrete cover is increased to $20 \mathrm{~mm}$, adhesion force reduced to $188.02 \mathrm{~N}$ and $248.17 \mathrm{~N}$ respectively. So this is a reduction of $39.84 \%$ for layout 1 and $45 \%$ for layout 2 . The rate at which adhesion force falls as the rebar distance increases is marginally lower for layout 1 than layout 2. Moreover, the influence of weight of the additional magnets of N-N-S-N-N arrangement is cancelled out by generating higher adhesion force which means layout 2 will have higher $\prod$ ratio compared to layout 1 as fig. 16 . 


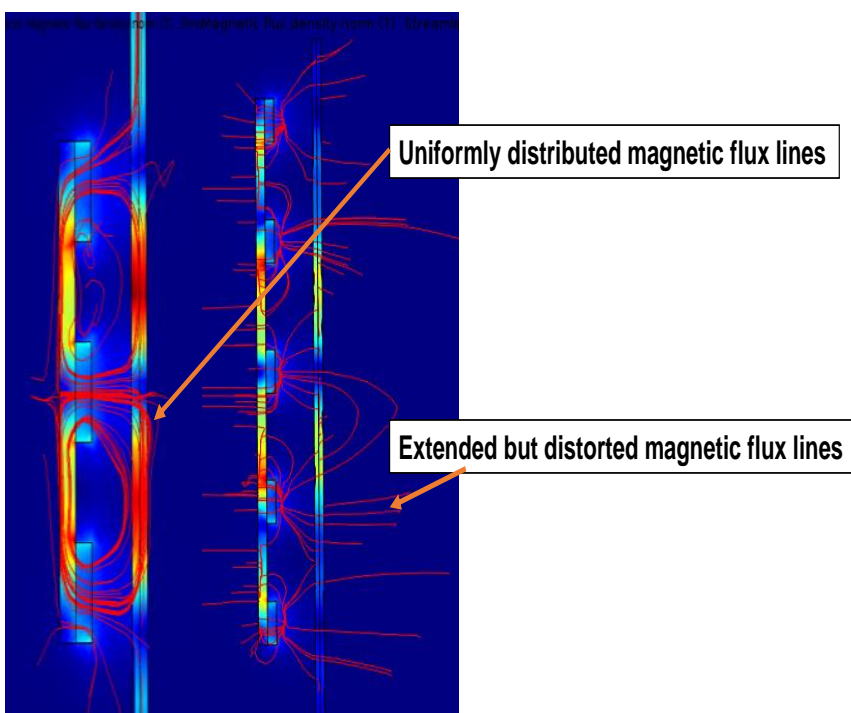

Fig. 14. Comparison of magnetic flux line propagation behaviour of layout 1 and layout 2 from left to right

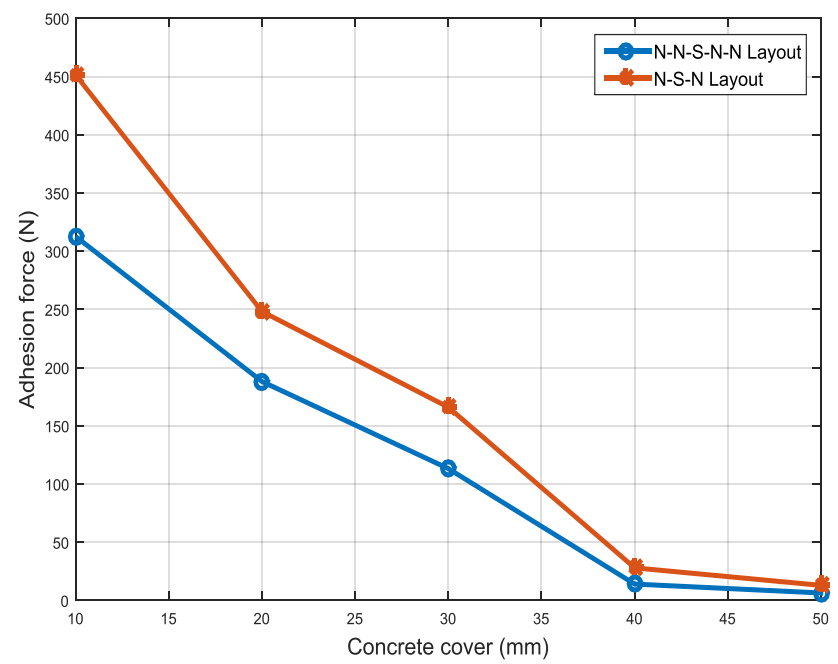

Fig. 15. Comparison of adhesion force between layout 1 and layout 2 for different concrete cover

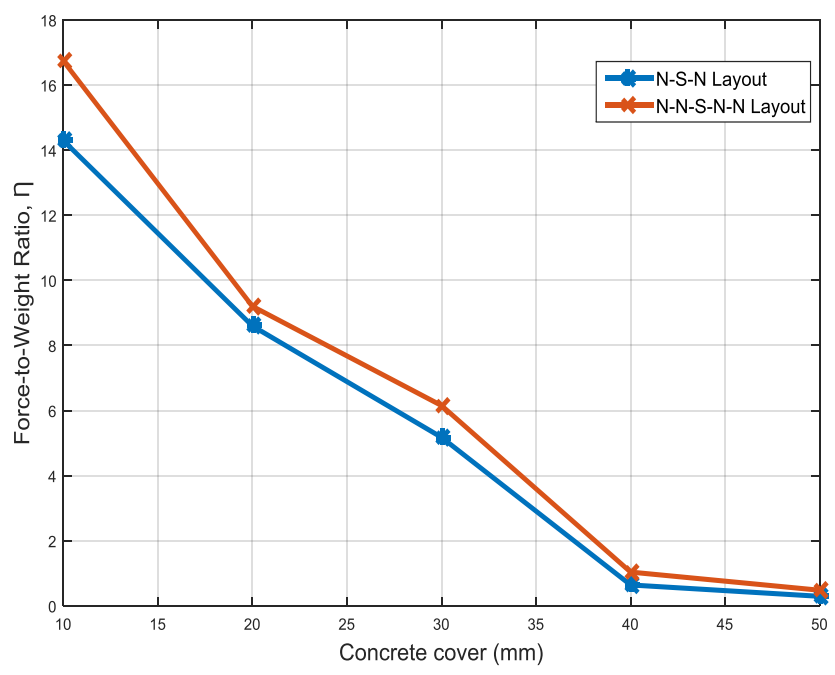

Fig. 16. Force to weight graph for two layouts

\section{EXPERIMENT SETUP AND RESULT DISCUSSION}

In order to validate the simulation results, a magnetic adhesion system consisting of three $\mathrm{N} 42$ grade neodymium magnets arranged in N-S-N orientation was built and attached to a prototype climbing robot. The measured dimensions of the whole system are: robot length $=360 \mathrm{~mm}$, robot width $=210$ $\mathrm{mm}$, height of the center of gravity $=15 \mathrm{~mm}$, magnet length and width $=50 \mathrm{~mm}$, magnet thickness $=10 \mathrm{~mm}$, yoke length $=$ $350 \mathrm{~mm}$. Two yokes with thickness of $5 \mathrm{~mm}$ and $15 \mathrm{~mm}$ as shown in figure 16 (a) were used for better comparison. The gap between the magnet surface and climbing surface is critical as a small gap increases the adhesion force significantly. Therefore, the gap was kept to a minimum of $2 \mathrm{~mm}$. The ability to pass obstacles has been considered to be of secondary importance since the aimed climbing structures are closely uniform. Each wheel is independently driven and a differential drive system is adopted to realize the turn. The wheel diameter is $63 \mathrm{~mm}$. Rubber wheel with polyurethane layer is chosen to increase the traction. The output torque and rotation of the motor are $2.16 \mathrm{Nm}$ and $30 \mathrm{rpm}$ respectively. The maximum speed of the robot is 6 meter per minute. The robot's net weight is $2.23 \mathrm{~kg}$ and $3.68 \mathrm{~kg}$ when $5 \mathrm{~mm}$ and $15 \mathrm{~mm}$ thick yoke is used respectively. Therefore, the required force for sliding avoidance can be obtained as $46 \mathrm{~N}$ and $76 \mathrm{~N}$ for $5 \mathrm{~mm}$ and $15 \mathrm{~mm}$ yoked robot respectively by using equation 3 and 4 when acceleration, $a=0.5 \mathrm{~m} / \mathrm{s}^{-2}$ and wheel friction coefficient, $\mu=0.5$.

Two FlexiForce A201 force sensors, capable of measuring force up to $445 \mathrm{~N}$ was mounted underneath the base of the motors situated on the top right and bottom left corner of the robot. As the attraction area of the adhesion module is uniform, therefore by taking the average value of the two sensors will give the actual adhesion force at a given test point. A 10-bit analogue- to-digital AVR microcontroller based embedded system module as shown in fig. 17 was used to capture and serial transmission of the analogue data and MATLAB was used for data analysis. An operator can access the on board control module via Bluetooth wireless communication system. Furthermore, the on- board devices are powered by four $1.5 \mathrm{~V}$ Lithium-ion Polymer (Li-Po) batteries that supply enough operating voltage for the motors and force measurement devices. As a result, the robot is totally umbilical free. The adhesion force of the prototype robot was measured for different yoke thickness and different rebar distance. Measurements were taken at three different test points along the height of a concrete column and the average value was taken for consideration. Fig. 18 shows a working prototype of the robot climbing a concrete wall of a building. The concrete cover was measured to be $20 \mathrm{~mm}$ using a rebar detector. Meanwhile, experiments of the wall climbing robot carrying a magnetic field measurement sensor and climbing a concrete column of a multistoried building also carried out. Weight of the magnetic hall-effect sensor and control unit was measured to be $1.6 \mathrm{~kg}$. The maximum adhesion force was measured $181.08 \mathrm{~N}$ using a $10 \mathrm{~mm}$ thick yoke.

Experiment vs simulation results shows when $5 \mathrm{~mm}$ thick yoke was used, the adhesion force reduced from $162.06 \mathrm{~N}$ to $4.0221 \mathrm{~N}$ as the concrete cover changed from $20 \mathrm{~mm}$ to 50 $\mathrm{mm}$. Using a $10 \mathrm{~mm}$ thick yoke produced slightly higher 
adhesion force for concrete cover from $20 \mathrm{~mm}$ to $35 \mathrm{~mm}$. However, if concrete cover is bigger than $35 \mathrm{~mm}$, then adhesion force for $10 \mathrm{~mm}$ thick yoke decrease sharply compared to $5 \mathrm{~mm}$ thick yoke as fig. 19 (a) and (b).

Adhesion force for different yoke thickness at a constant concrete cover of $30 \mathrm{~mm}$ also followed the same results achieved in simulations. For $5 \mathrm{~mm}$ thick yoke, the adhesion force was $121.26 \mathrm{~N}$ which increased to $160.17 \mathrm{~N}$ as the thickness was increased to $40 \mathrm{~mm}$. However, increased weight of the yoke had a reverse relationship to the force-to-weight ratio. Fig. 20 (b) shows a gradual fall of $\prod$ from 7.413 to 0.747 as the yoke thickness increased from $20 \mathrm{~mm}$ to $50 \mathrm{~mm}$. Therefore, yoke thickness of $20 \mathrm{~mm}$ should be an optimum design trade-off.
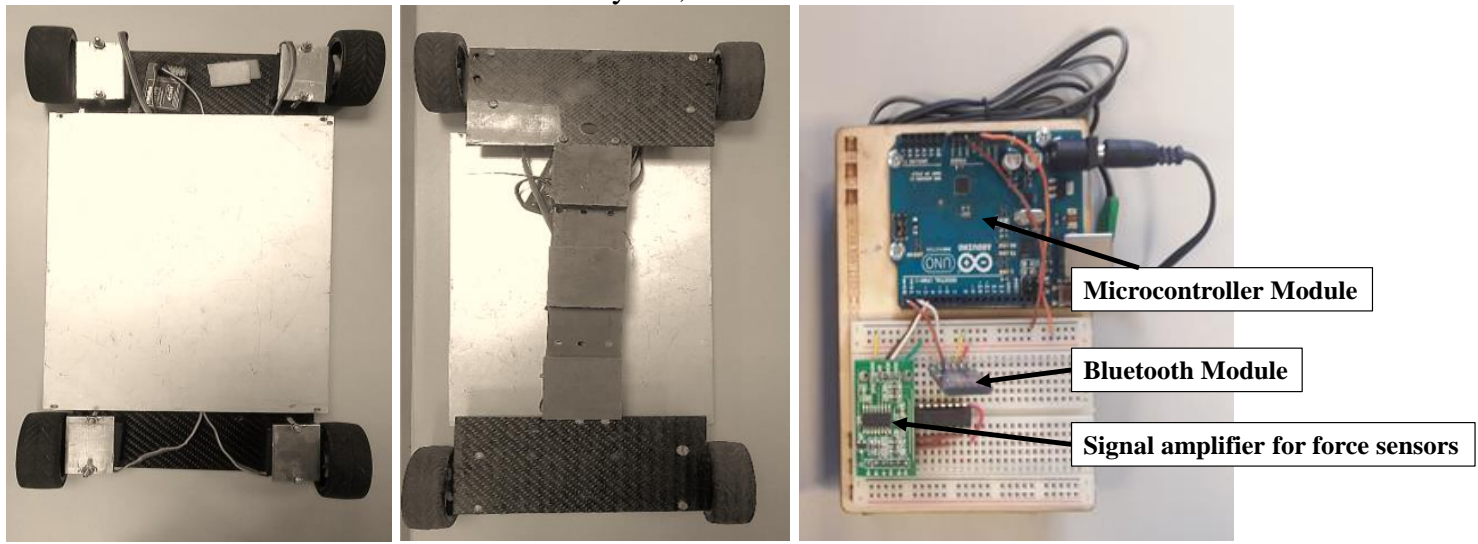

Fig. 17. Prototype of the concrete wall-climbing robot (top and bottom view) and adhesion force measurement unit circuit
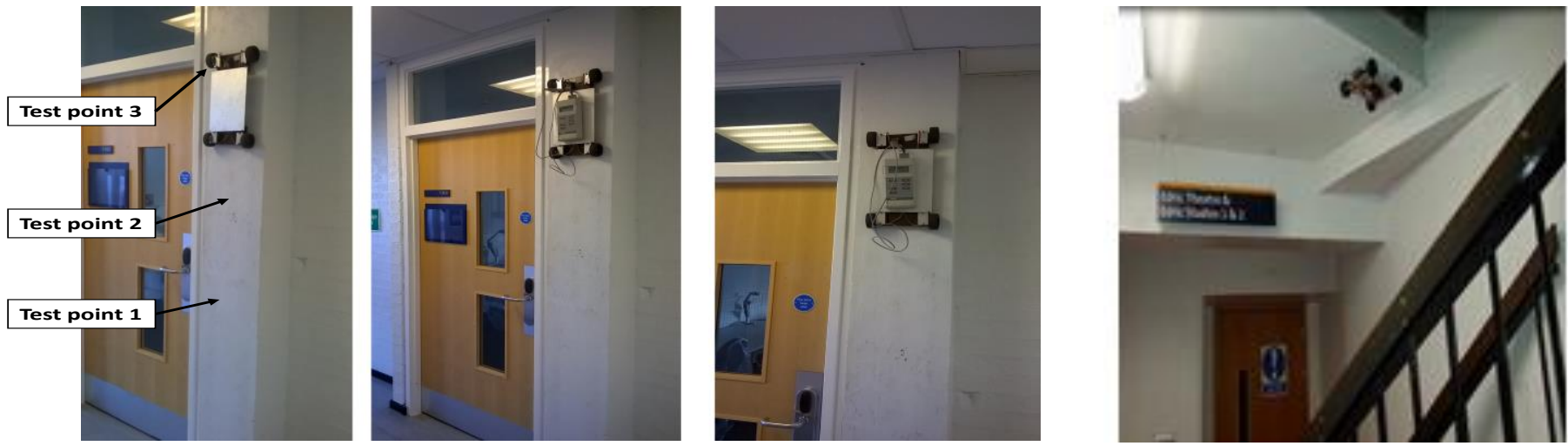

Fig. 18. Prototype robot climbing concrete column and ceiling with on board magnetic field sensor
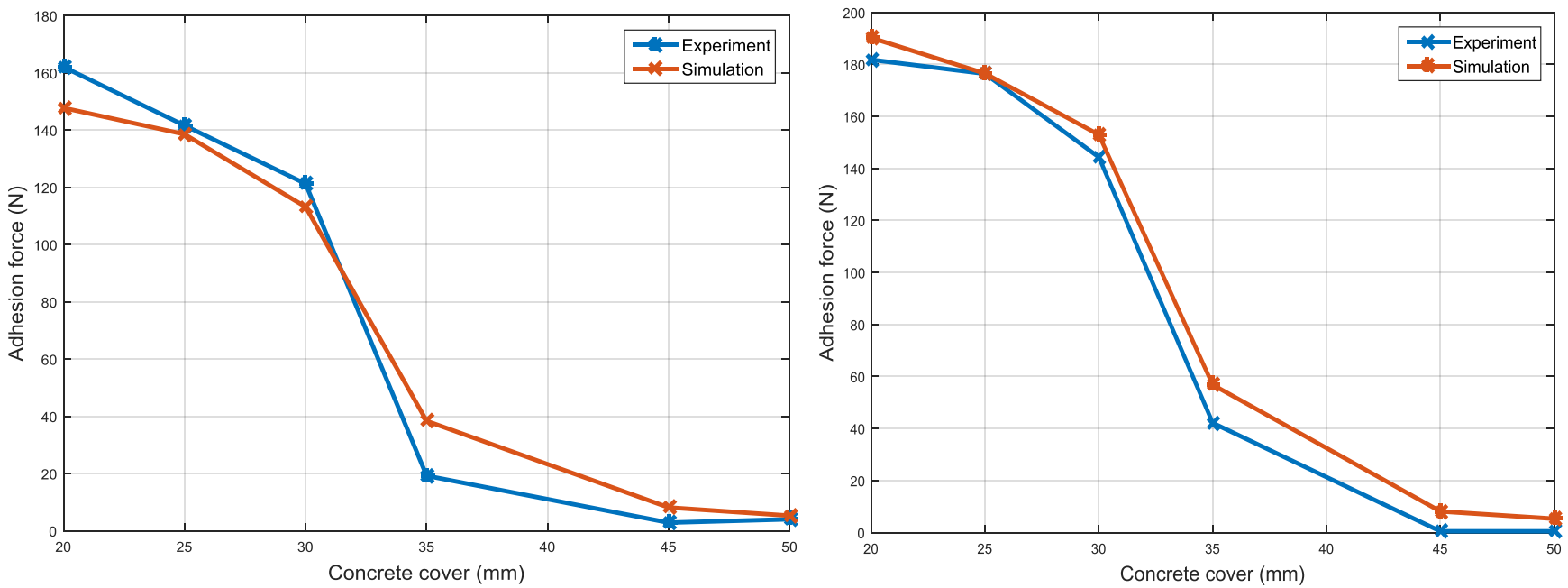

Fig. 19. Comparison of results at different concrete cover for (a) $5 \mathrm{~mm}$ thick yoke; (b) $10 \mathrm{~mm}$ thick yoke 

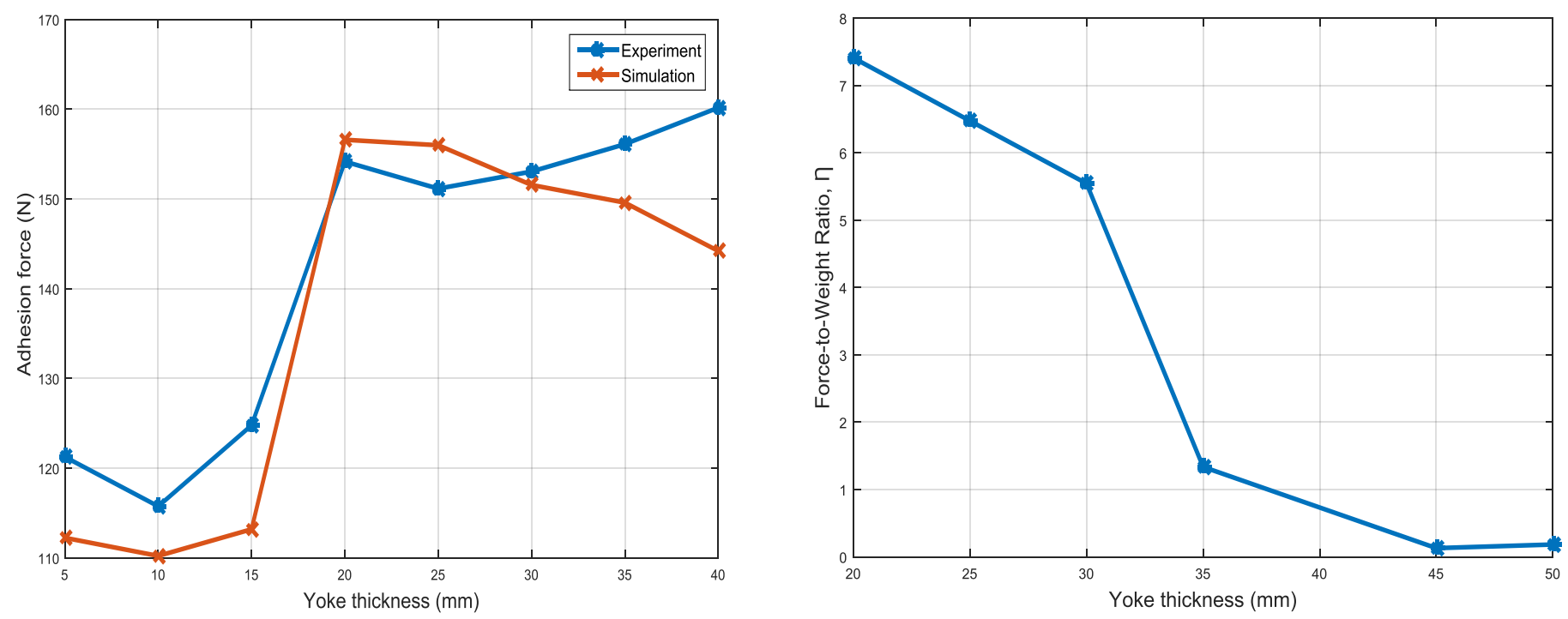

Fig. 20. (a) Experiment vs Simulation results for different thickness of yoke; (b) Experiment results for Force-to-Weight ratio, $\eta$ for different yoke at 30mm concrete cover

\section{COMPARISON STUDY}

This work is proposing permanent magnets based adhesion mechanism for concrete surface climbing robot. There are several literatures could be found that use permanent magnets mainly for ferrous wall climbing and other biological models such as adhesive legs or nano-spike gecko-bots for concrete wall climbing. However, those systems are not still suitable for real world application. Moreover, many of those robots are bulky, resulting in low force-to-weight ratio, $\eta$. A comparison study is of this proposed robot with other literature found is presented in table III.

TABLE III. COMPARISON OF THE PROPOSED RoBOtics AdHESION SYSTEM WITH OTHER SYTEMS FOUND IN THE LITERATURE

\begin{tabular}{|l|l|l|l|}
\hline & Ref [7] & Ref [8] & $\begin{array}{l}\text { This } \\
\text { work }\end{array}$ \\
\hline Robot weight (kg) & 30 & 18 & 2.23 \\
\hline $\begin{array}{l}\text { Maximum applicable } \\
\text { adhesion force (N) }\end{array}$ & 1400 & 667 & 121.26 \\
\hline Force-to-weight ratio & 4.76 & 3.77 & 5.54 \\
\hline Adhesion mechanism & Magnetic & Magnetic & Magnetic \\
\hline Locomotion & Wheel & Track & Wheel \\
\hline Application medium & $\begin{array}{l}\text { Ferrous } \\
\text { wall }\end{array}$ & $\begin{array}{l}\text { Ferrous } \\
\text { wall }\end{array}$ & $\begin{array}{l}\text { Concrete } \\
\text { wall }\end{array}$ \\
\hline
\end{tabular}

\section{CONCLUSION}

A novel technique of magnetic flux concentration mechanism has been presented. In contrast to existing research findings, this research aims to manipulate physical and material constrains to apply such adhesion system on a concrete surface where the attraction medium for permanent magnets is limited. The simulation results shows that the distance between the magnet and buried rebar has the maximum impact on the total adhesion force. The prototype system exhibits good payload capacity and can generate adhesion force of $121.26 \mathrm{~N}$ for the robot to climb a concrete wall reinforced with only one rebar located as far as $30 \mathrm{~mm}$. Considering the size and weight of the robot, a high payload capacity has been achieved by optimizing key design parameters of the adhesion mechanism. Overall, this mobile robot exhibits very good performance and force-toweight ratio compared to other reported permanent magnet based robotic systems. Though $12 \mathrm{~mm}$ diameter rebar was used for simulations, rebars used is much thicker $(55 \mathrm{~mm}$ in some cases) in its target application such as nuclear power plant and bridge column. Therefore, the adhesion force will be much higher in those conditions. Moreover, nearby rebars in a rebar mesh will also increase the adhesion force. To turn the robot into a practical application, a higher grade (N52) of neodymium magnets could be used that will increase adhesion while keeping the force to weight ratio as same as N42 grade magnets. Nonetheless, the proposed methodology will be the foundation of further research. Future research will involve investigating the effects of a rebar mesh on adhesion force as well as carrying out further testing in real life applications.

\section{REFERENCES}

[1] GSSI Case study, "A Tall Order: Scanning a 300-feet Chimney", Geophysical Survey Systems, Inc., Salem, USA, 2009.

[2] B. Zhiqiang, G. Yisheng, C. Shizhong, Z. Haifei, and Z. Hong, "A Miniature Biped Wall-Climbing Robot for Inspection of Magnetic Metal Surfaces", IEEE International Conference on Robotics and Biomimetics, 2012, Guangzhou, China.

[3] T. Sattar, E. Leon-Rodriguez Hernando, S. Jianzhong, "Amphibious NDT Robots", Climbing and Walking Robots, Towards New Applications, International Journal of Advanced Robotics Systems, Chapter 6, pp. 24, 2007.

[4] W. Fischer, F. Tache, G. Caprari, and R. Siegwart, "Magnetic wheeled robot with high mobility but only 2DOF to Control", International Conference on Climbing and Walking Robots, 2008, Portugal.

[5] F. Tache, W. Fischer, G. Caprari, R. Moser, F. Mondada, R. Siegwart, "Magnebike: a magnetic wheeled robot with high mobility for inspecting complex shaped structures", Journal of Field Robotics, Vol. 26, pp. 453-76, 2009.

[6] T. Kang, H. Kim, T. Son, H. Choi, "Design of quadruped walking and climbing robot"; IEEE/RSF International Conference on Intelligent Robots and System, 2003, Vol. 1, pp. 619-24.

[7] J. Shang, B. Bridge, T. Sattar, S. Mondal, and A. Brenner, "Development of a climbing robot for inspection of long weld lines", Industrial Robot, Vol. 35, No. 3, pp. 217-223, 2008. 
[8] P. Kalra, G. Jason, M. Max, "A wall climbing robot for oil tank inspection", IEEE International Conference on Robotics and Biomimetics, China, 2006, pp. 1523-1528.

[9] L. Briones, P. Bustamante, M. Serna, "ROBICEN: a wall-climbing pneumatic robot for inspection in nuclear power plants", Robotics and Computer-Integrated Manufacturing, pp. 287-292, 1994.

[10] A. Nagakubo, S. Hirose, "Walking and running of the quadruped wallclimbing robot", IEEE International Conference on Robotics and Automation, pp.1005-1012, 1994.

[11] R. Pack, J. Christopher, K. Kawamura, "A Rubbertuator-based structureclimbing inspection Robot", IEEE International Conference on Robotics and Automation, pp. 1869-1874, 1997.

[12] X. Chen, M. Wager, M. Nayyerloo, W. Wang, J. Chase, "A novel wall climbing Robot based on Bernoulli effect", International Conference on Mechatronic and Embedded Systems and Applications, Beijing, China, 2008 .
[13] S. Tso, T. Feng, "Robot Assisted Wall Inspection for Improved Maintenance of High-Rise Buildings", International Symposium on Automation and Robotics in Construction, China, 2001, pp 63-71.

[14] D. Fanella, "Design of Low-Rise Reinforced Concrete Buildings Based on 2009 IBC", ASCE/SEI 7-05, ACI 318-08. International Code Council, Washington, DC.

[15] European Standard. Eurocode 2, "Design of Concrete Structures, General Rules".

[16] W. Xilin, J. QIU, T. Junyong, "Analytical dynamics and application in Electromechanical System", Magazine of Science press, Beijing, pp. 212-213, 2003.

[17] P. Yao, D. Li, "The magnetic field analysis and optimization of permanent-magnetic adhesion device for a novel wall-climbing robot," Proc. of the IEEE Conference on International Technology and Innovation, pp. 2-6, 2009. 